\title{
Bendecir para saludar en Plauto. Redistribución de la función pragmática*
}

\author{
Łukasz Berger \\ Universidad Adam Mickiewicz de Poznań \\ lberger@amu.edu.pl \\ ORCID iD: http://orcid.org/0000-0002-8194-4681 \\ Bleesing for a greeting in Plautus. \\ Redistribution of a pragmatic function
}

\begin{abstract}
Este artículo se ocupa de la redistribución de una función pragmática, esto es, repragmaticalización (fenómeno también conocido como «discursivización») de las fórmulas latinas de bendición. La repragmaticalización de estas expresiones consistió en su reinterpretación como expresiones de agradecimiento rutinizadas, para, finalmente, funcionar como unas fórmulas de saludo (di tibi dent quae uelis!; di te ament!). En primer lugar, se lleva a cabo un análisis de los comienzos de diálogo que incluyen estas expresiones. Además, el artículo ofrece algunas referencias adicionales al sistema romano de la retribución compensatoria, que ha sido relacionado con las rutinas comunicativas de agradecer y saludar. A modo de conclusión, se ofrecen algunas reflexiones sobre los procesos de repragmaticalización.
\end{abstract}

Palabras clave: bendición; agradecimiento; saludo; fórmula conversacional; pragmaticalización;
The paper focuses on the redistribution of pragmatic function, repragmaticalization (the phenomenon also known as discursisation). It is assumed that the Latin blessing formulae, as reproduced in the comedies by Plautus, underwent discursisation that resulted in its reinterpretion as a routinized expression of gratitude, and, finally, as greeting formulae (di tibi dent quae uelis!; di te ament!). Firstly, an analysis of the dialogue openings that include this particular wording of the salutatio ritual is conducted. Moreover, the study offers additional insights into the Roman reciprocal donation system, which has been related to the communicative routines of thanking and greeting. In conclusion some general remarks are made about the nature of the repragmaticalization process and its results.

Key words: blessing; thanks; greeting; conversational formula; pragmaticalization; repragma-

* El artículo forma parte del proyecto de investigación «Estructura de la conversación en la comedia romana frente a la cortesía lingüística», financiado por el Ministerio de Ciencia y Educación Superior polaco (1314/MOB/IV/2015/0). Asimismo, el autor expresa su gratitud a dos Informantes anónimos cuyas sugerencias y correcciones han contribuido a la calidad del presente trabajo.

Copyright: (C) 2017 CSIC. Este es un artículo de acceso abierto distribuido bajo los términos de la licencia de uso y distribución Creative Commons Attribution (CC-by) España 3.0. 
repragmaticalización; Plauto; di te ament!; di tibi dent quae uelis!

ticalization; Plautus; di te ament!; di tibi dent quae uelis!

Cómo citar este artículo / Citation: Berger, Łukasz 2017: «Bendecir para saludar en Plauto. Redistribución de la función pragmática», Emerita 85 (2), pp. 261-287.

\section{INTRODUCCIÓN}

Las fórmulas di te ament! y di tibi dent quae uelis! pertenecen a un nutrido grupo de expresiones de carácter religioso que, como resultado de su uso mecanizado, llegaron a desarrollar funciones discursivas diferentes a la esperable de su semántica. Ejemplos de este fenómeno son la fórmula ita me di ament!, que intensifica la fuerza ilocutiva asertiva (Amph. 597: Sos. ... neque, ita me di ament, credebam primo mihimet Sosiae $)^{1}$, o, en enunciados enfocados hacia el destinatario, el sintagma si te di ament, que aparece como refuerzo en avisos o recomendaciones irónicas, que pueden esconder una amenaza (Mil. 571: Per. Ne tu hercle, si te di ament, linguam comprimes). En estas fórmulas, cuyo sentido propio las vincula en origen con las creencias de los hablantes ${ }^{2}$, el ritual propiamente religioso (de bendecir, maldecir, invocar a la deidad, etc.) ha sido sustituido, a través de un proceso de pragmaticalización ${ }^{3}$, por un valor comunicativo muy fijado (saludar, aseverar, amenazar ...) que permite interpretarlas como fórmulas conversacionales ${ }^{4}$. Claridge y Arnovick (2010, pp. 179-182), dan cuenta de los mecanismos que están detrás de esta evolución: las fórmulas sufren una desemantización (parcial) y cierta fijación formal, a la par que cambian de categoría gramatical y empiezan a operar sobre otro ámbito del texto (todo el enunciado, un segmento del discurso, etc.).

1 Véase Griffe 1985.

${ }^{2}$ Véase Hanson 1959.

3 Al igual que la «gramaticalización», la «pragmaticalización» no es un término (ni un fenómeno) entendido de manera unánime. Aquí seguimos, a grandes líneas, el marco metodológico de Claridge y Arnovick 2010. En la discusión acerca de las diferencias entre la gramaticalización y la pragmaticalización, sigue siendo crucial la definición de la gramática misma. Según indican Degand y Evers-Vermeul 2015, los lingüistas que distinguen el nivel pragmático-textual de los fenómenos tradicionalmente adscritos al nivel gramatical suelen apostar por la pragmaticalización como un proceso aparte.

${ }^{4}$ Entendemos las fórmulas conversacionales (denominación intercambiable en este trabajo por «rutinas» y «clichés lingüísticos») según la definición amplia de Coulmas 1981a, pp. 2-3: «... highly conventionalized prepatterned expresions whose occurence is tied to more or less standardized communication situations». 
Lo más destacable es que la pragmaticalización genera una nueva función y un nuevo significado, derivados no tanto del contenido como del contexto de uso y las intenciones del hablante.

Entre las fórmulas de origen religioso más comunes se puede mencionar las bendiciones y las maldiciones, como tibi di faciant bene! (p. ej. Mil. 570, Persa 488) y di te perdant! (p. ej. Epid. 23, Merc. 967), respectivamente. En este trabajo, nos ocuparemos de las primeras, que suelen asumir más de una función pragmática y discursiva ${ }^{5}$. Cabe destacar en primer lugar los casos con predominio del significado léxico, es decir, las bendiciones entendidas propiamente como actos desiderativos. Así se interpretan las palabras de Pséudolo dirigidas a Simia (1a-b), pese a estar teñidas de una fuerte ironía:

(1a) Simi. Te quoque etiam dolis atque mendaciis, / qui magister mihi es, antidibo, ut scias. /

Pseu. Iuppiter te mihi seruet.

Simi. Immo mihi. / sed uide, ornatus hic me satin condecet? /

Pseu. Optume habet.

Simi. Esto. / (Pseud. 932-935a $)^{6}$

(1b) Pseu. Tantum tibi boni di immortales duint quantum tu tibi optes; / nam si exoptem, quantum dignu's tantum dent, minus nihilo sit. / neque ego hoc homine quemquam uidi magis malum et maleficum. / Simi. Tun id mi? Pseu. Taceo. (Pseud. 932-938a)

(1c) PER. Et ego impetrare dico id quod petis. PAL. At te Iuppiter / bene amet (Mil. 231-232)

${ }^{5}$ La función pragmática se entiende aquí como el significado que cobra una expresión lingüística a través de su uso dentro de la interacción verbal. A nivel interpersonal, por tanto, puede equivaler a la fuerza ilocutiva. A nivel de la estructuración del discurso, ciertas expresiones lingüísticas desempeñan, además, una función discursiva (su apertura y cierre, cambios de turno, etc.), que tratamos como un subtipo de las funciones pragmáticas. Los saludos, consecuentemente, desempeñarían una doble función: la de marcar el inicio del diálogo y la de un acto expresivo, según la tradicional categorización de Searle (véase Ronan 2015, pp. 27-32).

${ }^{6}$ Los textos plautinos reproducidos en este trabajo siguen la edición de De Melo 20112013. Prescindimos de la indicación del autor en la referencia de las comedias. 
Como acabamos de ver, dentro de la unidad dialógica mínima (el par adyacente $)^{7}$, la bendición puede tomar tanto una posición iniciativa (1b), como de réplica (1a). Además, en posición reactiva la bendición, de acuerdo con Unceta Gómez 2010, p. 627, es frecuentemente empleada como una rutina lingüística para expresar gratitud (1c) $)^{8}$, siendo este un fenómeno presente en algunas lenguas europeas modernas (p. ej. esp. que Dios te bendiga / te lo pague; pol. Bóg zapłać [«que Dios pague»] = «gracias»).

Ahora bien, dos fórmulas de bendición (di te ament! y di tibi dent quae uelis!) adquieren también en el corpus plautino una función discursiva adicional: la de saludos, que, aparte de constituir actos expresivos ${ }^{9}$, permiten la apertura del diálogo. Esta supuesta redistribución de la función pragmática (a través de adquirir una función discursiva) tampoco resulta extraña si la comparamos con ciertas evoluciones paralelas en las lenguas europeas: las fórmulas de bendición son empleadas actualmente por algunas comunidades de hablantes bien para saludar (alem. austr. Grüss Gott!), bien como despedida (ing. good-bye! < God be with ye!, esp. jadiós! < ; a Dios [te encomiendo]!). En el caso del latín plautino, el empleo de las fórmulas di te ament! y di tibi dent quae uelis! en la apertura dialógica, tal como intentaremos demostrar en las páginas que siguen, está supeditado a ciertos rasgos de mentalidad romana. En esta premisa seguimos las pautas metodológicas de Coulmas:

... a routine is not an expression of strategy, but rather an expression which is appropriate to a situation of a certain kind or a strategy which is appropriate relative to certain communicative ends. Hence routines must be accounted for together with the description of situational or institutional context within which they could be produced. (Coulmas 1981a, p. 16)

7 De acuerdo con el Análisis Conversacional, el par adyacente (prototípico) es un par de enunciados producidos por diferentes interlocutores y convencionalmente entrelazados (p. ej., el par adyacente «pregunta - respuesta»). El sistema de pares de adyacencia constituye el principal mecanismo de gestión de los cambios de turnos (Levinson 1983, p. 303-304). Sobre la aplicación de los principios del Análisis Conversacional al latín, y en concreto al diálogo plautino, véase Monserrat Roig 2015, pp. 136-141 (con bibliografía).

${ }^{8}$ Véanse Asin. 654 (di te servassint semper), Trin. 384 (di te servassint mihi).

${ }^{9}$ Ferguson 1981 presenta algunas generalidades sobre las fórmulas de saludo y su uso en la fase inicial del diálogo. Duranti 1997, pp. 64-67, resume brevemente los estudios sobre la apertura dialógica desde el punto de vista de diferentes metodologías. Pavlidou 2014, pp. 357-363, recoge la principal bibliografía del Análisis Conversacional dedicada a este tema hasta la fecha. 
Consiguientemente, si una misma fórmula conversacional aparece con una función pragmática diferente de la propia e incluso en una posición dentro de la cadena dialógica diferente de la que suele ocupar, este proceso se ha de explicar a través de los elementos de la nueva situación comunicativa que han causado que esta expresión lingüística sea también funcional y apropiada fuera de su entorno acostumbrado. Dicho de otra manera, lo institucional nos servirá para arrojar algo de luz sobre lo situacional ${ }^{10}$.

\section{RECIPROCICIDAD, INTERCAMBIOS Y RETRIBUCIONES}

Por lo general, los dioses mencionados en las fórmulas de origen sacral arriba presentadas realizan una función intermediadora entre los participantes del acto comunicativo; a saber, son instrumentos de la sanción socio-religiosa implícita en actos de habla como juramentos (ita me di ament!), amenazas (si te di ament), maldiciones (di te perdant!) o bendiciones (di tibi faciant bene / dent quae uelis!, di te ament!). De tal manera, el favor o la hostilidad divinos se conciben como un «objeto» de transacción discursiva, la base para crear y negociar, a lo largo del diálogo, las relaciones interpersonales entre los hablantes. Un momento crítico del proceso comunicativo es, sin duda, la apertura dialógica, cuya parte formularia no solamente facilita el paso a la fase media ${ }^{11}$, sino que también aporta información sobre las imágenes sociales de los interlocutores ${ }^{12}$. Los papeles que los participantes negocian durante el saludo, a su vez, determinan el tipo de interacción que irán desarrollando.

${ }^{10}$ La parte analítica del presente trabajo se basa en el estudio cuya temprana versión (en polaco) fue incluida en nuestra tesis doctoral (Berger 2016, pp. 145-169). Aquí estos datos han sido reinterpretados a la luz de la teoría de la cortesía lingüística y el fenómeno de la repragmaticalización. Para la redacción final, muy valiosos resultaron los comentarios del Prof. Luis Unceta Gómez, a quien expresamos nuestro sumo agradecimiento.

${ }^{11}$ El diálogo suele dividirse en tres partes: inicial, media y final (Laver 1975, Pavlidou 2014). La apertura dialógica son los primeros pares de adyacencia que (prototípicamente) incluyen formas de establecer contacto (ing. summons), la identificación de los hablantes, el intercambio de saludos, el tema preliminar (ing. small talk, cf. infra, n 37) y varios mecanismos de transición a la parte media. Para la apertura dialógica en Plauto véase Hoffman 1983, Berger 2016.

12 Nos referimos aquí al concepto de «imagen social» (ing. face) desarrollado y definido por Goffman 1955 , p. 213 , como «the positive social value a person effectively claims for himself by the line the others assume he has taken during a particular contact». 
Sirva de ilustración la siguiente escena de salutatio (2) entre un joven y un lenón:

(2) Ago. Saluos sis, leno.

LyC. Di te ament, Agorastocles. /

Ago. Magis me benigne nunc salutas quam antidhac. (Poen. 751-752)

Las formas lingüísticas utilizadas por el lenón Lico (la fórmula di te ament! junto con un tratamiento nominal) despiertan la desconfianza de su interlocutor, quien se esperaba, al parecer, una apertura dialógica algo más neutra o, por lo menos, no tan marcadamente cortés. Así, Agorastocles ha sido capaz de diagnosticar la actitud y las intenciones comunicativas del lenón incluso antes de empezar el diálogo propiamente dicho: Lico iba a conseguir sus objetivos conversacionales a través de una cortesía estratégica que, dadas las circunstancias del encuentro, a ojos de su interlocutor ha parecido ir más allá del simple ritual de saludo ${ }^{13}$. Gracias al comentario metapragmático de Agorastocles ( $m a-$ gis me benigne nunc salutas...), llegamos a saber que uno de los elementos que no esperaba en la réplica del lenón es la fórmula de bendición, aparentemente algo más elevada y cortés que un simple salue!

Mucho más armónica y recíproca ha de ser considerada la escena de $s a$ lutatio entre dos esclavos, Tóxilo y Sagaristión, que parecen parodiar el lenguaje amistoso de sus amos ${ }^{14}$ :

(3) Tox. O Sagaristio, di ament te.

SAG. O Toxile, dabunt di quae exoptes. / Vt uales?

Tox. Vt queo.

SAG. Quid agitur?

Tox. Viuitur. (Persa 16-17)

Ambos interlocutores no solamente se devuelven el saludo, sino que también responden a cada acto conversacional con formas lingüísticas equivalentes: la simetría comunicativa va paralela a la simetría de las relaciones interpersonales.

${ }^{13}$ Atendemos a la distinción propuesta por Watts (2003) entre la cortesía propiamente dicha (ing. polite behaviour) y el comportamiento lingüístico «político», que consiste meramente en cumplir con las obligaciones socioculturales (ing. political behavior).

${ }^{14}$ Compárense, en este contexto, sobre todo las aperturas dialógicas amistosas entre dos senes en Merc. 283-301, Trin. 48-67. 
Si atendemos ahora a la caracterización lingüística de las fórmulas de origen sacral, hay que destacar el uso recurrente de los verbos dare y amare, que codifican las conceptualizaciones romanas de sus prácticas religiosas. Al analizar la fórmula de juramento ita me di ament!, Griffe 1985, pp. 294-295, arguye que este verbo amare ha de tener un significado algo más especializado que evoca la reciprocicidad en las relaciones entre los dioses y los creyentes: la benevolencia divina a cambio de la devoción humana. De ahí que el amare romano (distinto de nuestra noción del 'amar' cristiano o romántico) no se aleje demasiado de la idea del intercambio explicitada en las fórmulas que contienen el verbo dare. En consecuencia, el antiguo concepto romano de amicitia, la base de las relaciones sociales, presupone un sistema basado en prestaciones que llegó a constituir una red de expectativas mutuas (cf. Verboven 2011, pp.411-412). Como ilustración de esta idea, veamos una cómica conversación entre Deméneto y su esclavo, Líbano, donde a la cadena dialógica se añade una cadena de favores:

(4) LiB. Vsque ad mortem uolo. /

Dem. Caue sis malam rem.

Liв. Vxoris dico, non tuam. /

Dem. Dono te ob istuc dictum, ut expers sis metu. /

LiB. Di tibi dent quaequomque optes.

Dem. Redde operam mihi. (Asin. 42-46)

Cuando el frívolo siervo menciona la muerte, su interlocutor lo interpreta como algo ominoso, hasta que Líbano neutraliza su propia maldición convirtiendo sus palabras en un buen deseo: se autocorrige matizando que se refiere a la muerte de la mujer de su amo. A cambio de esta evidente «deferencia», Deméneto promete ( $\underline{\text { dono }}$ te) librar a Líbano del castigo. El agradecido esclavo, a su vez, pronuncia la fórmula gratulatoria di tibi dent quaequomque optes, evocando la futura recompensa divina, lo cual el anciano aprovecha como una buena ocasión para recaudar la deuda: Deméneto quiere que el interlocutor, a cambio, le preste ... atención (redde operam mihi). Esta breve secuencia dialógica ejemplifica muy bien el mencionado sistema romano de prestaciones recíprocas, descrito por Benveniste 1966, p. 322, como una de las instituciones de las sociedades indoeuropeas: los bienes recibidos (munus) conducen a la obligación (onus) de recompensar- 
los con el fin de restaurar el equilibrio de poderes. Consiguientemente, la cadena de favores (expresiones de beneuolentia) y retribuciones (expresiones de gratia) entre Deméneto y Líbano podría ser infinita si no fuera por la fórmula gratulatoria ${ }^{15}$. Hacer que los dioses intervengan en el sistema de prestaciones tiene una ventaja desde el punto de vista de la interacción: el anciano promete a su esclavo un buen tratamiento, mientras que este se lo recompensa con una bendición, según la lógica: «me has dado tú: que te lo devuelvan los dioses». Es aquí donde Deméneto, en contra de las convenciones de cortesía, prolonga la cadena de los favores e insiste en que su deuda sea saldada por el mismo Líbano hic et nunc: «yo te he dado, devuélveme tú (ahora)».

La apertura dialógica como acto que entraña un riesgo para la imagen tanto del iniciador del contacto como para la de su interlocutor, necesita de estrategias comunicativas que, desde el estudio clásico de Brown y Levinson 1987, se conocen con el nombre de cortesía positiva (apreciativa) ${ }^{16}$. El recurso más convencional es declarar beneuolentia hacia el interlocutor, deseándole buena salud, como forma de invitar a entablar la conversación. No podemos obviar, por tanto, que el saludo prototípico es un acto desiderativo (p. ej. salue!, saluos, -a sis!), sobre cuya base podrían forjarse fórmulas con verbos performativos (te saluto!, te iubeo saluere!). En cualquier caso el saludo, normalmente, implica otro saludo (contrasaludo), con el que forma un par de adyacencia, pero, como vemos en (5), a veces basta con que el saludado exprese su gratitud:

(5) Lyc. [...] hospes hospitem / salutat. saluom te aduenire gaudeo. /

Col. Multa tibi di dent bona, quom me saluom esse uis. (Poen. 685-687)

Pese a que la réplica de Colibisco no puede ser considerada un saludo propiamente dicho, deja entrever la motivación del hablante para emplear en esta posición la fórmula de bendición multa tibi di dent bona! Es interesante notar que el saludado explicita la razón que le ha llevado a realizar un

${ }^{15}$ Cf. Sen., Ben. II 18.5: ... nihilo minus etiam relata gratia cohaeremus; debeo enim, cum reddidi, rursus incipere. Para un análisis de la amicitia en Plauto, entendida como un intercambio de favores, véase Burton 2004.

${ }^{16}$ La cortesía positiva está relacionada con las necesidades del hablante de ser apreciado por su interlocutor, por ejemplo, a través del reconocimiento de la imagen social positiva que este va creando a lo largo de la interacción (Brown y Levinson 1987, p. 70). 
acto gratulatorio ${ }^{17}$ : este es el esquema canónico del agradecimiento en el lenguaje plautino ${ }^{18}$. De ahí que consideremos (5) un caso puente en la evolución que estamos comentando: el acto de gratitud se solapa, en dicho par adyacente, con la reacción convencional a la salutatio, es decir, el contrasaludo. Si ahora volvemos a la iniciación del diálogo de (2) y (3), entenderemos mejor el mecanismo que ha provocado la redistribución de la función pragmática de las bendiciones di te ament! y di tibi dent quae uelis! como fórmulas de saludo. Significativamente, aparte de la escena de salutatio de (5), en ningún otro ejemplo de apertura dialógica dichas expresiones necesitarán de una justificación para tal acto de habla. Por último, cabe concluir que, gracias al empleo de fórmulas especializadas en el acto de habla gratulatorio, se destaca (y, en el caso de di tibi dent quae uelis!, se explicita) la conceptualización de par adyacente del saludo como un intercambio de prestaciones (munus-onus) ${ }^{19}$.

La Tabla 1 ilustra las posibles interrelaciones de los actos que subyacen a la apertura dialógica: el acto optativo (la bendición) constituye la base del acto gratulatorio, mientras que este último, en ciertas posiciones, puede ser reinterpretado como un contrasaludo. Este proceso resulta aún más diáfano, si recordamos que el saludo (iniciativo) también deriva de un contexto desiderativo. De ahí que sea más fácil explicar la equivalencia resultante entre el imperativo fosilizado salue! («que tengas salud») y las bendiciones («que recibas la benevolencia de los dioses»).

17 Así es interpretado Poen. 687 en Unceta Gómez 2010, p. 627.

18 Véanse, por ejemplo, Poen. 207-209 (multa tibi di dent bona, / quom ...), 667-668 (di deaeque uobis multa bona dent, quom...) y Stich. 469 (bene atque amice dicis. di dent quae uelis), donde la justificación del agradecimiento se expresa de manera paratáctica. Según el estudio de Unceta Gómez 2010, p. 632, hacer hincapié en el favor prestado en lugar de en la deuda que siente el hablante es la estrategia de cortesía más utilizada en la comedia. Así, se evitan las sanciones sociales relacionadas con el sistema de prestaciones compensatorias (v. supra).

${ }_{19}$ Esta conceptualización de la comunicación no sería una cualidad exclusiva de la mentalidad romana. El sistema de dones y contradones fue relacionado, hace tiempo, con la institución del saludo por la etnógrafa Goody 1972, pp. 55-56, quien estudió la tribu gonja (habitantes del norte de Ghana). Asimismo, Coulmas 1981b, p. 87, localiza un patrón similar en la sociedad japonesa. 
Tabla 1. Par adyacente del saludo como sistema de prestaciones recíprocas.

\begin{tabular}{|c|c|c|c|c|}
\hline & iniciación & réplica & \multicolumn{2}{|c|}{ actos realizados } \\
\hline \multirow{2}{*}{$\begin{array}{c}\text { Locutor } 1 \\
\text { (quien saluda) }\end{array}$} & $\begin{array}{c}\text { salue } \\
\text { [velim] saluos, - a sis }\end{array}$ & & optativo & \multirow{2}{*}{ saludo } \\
\hline & $=$ tibi salutem do & & saludo & \\
\hline \multirow{3}{*}{$\begin{array}{l}\text { Locutor } 2 \\
\text { (quien es } \\
\text { saludado) }\end{array}$} & & $\begin{array}{c}\text { [velim] di tibi dent } \\
\text { quae uelis } \\
\text { [velim] di te ament }\end{array}$ & $\begin{array}{c}\text { optativo } \\
\text { (bendición) }\end{array}$ & \multirow{3}{*}{$\begin{array}{l}\text { respuesta al } \\
\text { saludo }\end{array}$} \\
\hline & & $=$ tibi gratias ago & gratulatorio & \\
\hline & & $=$ tibi salutem (red)do & $\begin{array}{l}\text { respuesta al } \\
\text { saludo }\end{array}$ & \\
\hline
\end{tabular}

A continuación, nos proponemos interpretar esta cadena de actos implícitos (secundarios) en las fórmulas del tipo di te ament! desde una perspectiva diacrónica, lo cual nos facilitará la posible motivación para la redistribución de su función pragmática: del acto optativo y gratulatorio hasta la respuesta al saludo. En los apartados que siguen, analizaremos los usos de dichas fórmulas trazando, por separado, sus particularidades situacionales.

\section{ANÁLISIS DE LAS FÓRMULAS DE SALUDO: LA BENDICIÓN}

Durante mucho tiempo, en las aproximaciones teóricas a los saludos (y la comunicación fática en general), dominó el prejuicio de que se trataba de signos lingüísticos desprovistos por completo de contenido proposicional ${ }^{20}$. Duranti 1997, p. 70, apunta con mucha razón que, a pesar de estar muy idiomatizadas y ser predecibles, las fórmulas de salutatio mantienen algo de su semántica, arraigado en el contexto de la interacción. El análisis que proponemos a continuación tiene el propósito de localizar los factores que subyacen en la elección por parte de los hablantes de las fórmulas de bendición (y sus variantes) como procedimiento de apertura dialógica.

En primer lugar, hemos de destacar que, dentro del corpus de las comedias de Plauto, el saludo es realizado con mayor frecuencia por medio de un enun-

${ }^{20}$ Véase Goffman 1971, p. 90, quien indicaba que los saludos se encuentran entre «the most conventionalized and perfunctory doings we engage in and traditionally have been treated by students of modern society as part of dust of social activity, empty and trivial». 
ciado de sentido desiderativo del tipo salue!, salueto! o saluos/ - a sis! ${ }^{21}$ : estas fórmulas, pese a conllevar diferentes significados interaccionales, aparecen en ambas posiciones del par adyacente, por lo cual deben considerarse como las formas más comunes. Por otra parte, expresiones algo más artificiosas como iube o te saluere! (Most. 568: TrA. ... saluere iubeo te, Misargyrides, bene)o Locutor $_{1}$ salutat Locutor ${ }_{2}$ ! (p. ej. Amph. 676: AмPH. Amphitruo uxorem salutat laetus speratam suam) funcionan únicamente como la primera posición del par. Frente a esto, las fórmulas de bendición, con algunas excepciones significativas, se encuentran exclusivamente en posición reactiva dentro de la pareja adyacente de la salutatio (véase Tabla 2). El resto de sus características se pondrá de relieve en el estudio contextual de sus testimonios.

1. Di tibi dent quae uelis!

A diferencia de las fórmulas de saludo más especializadas y automatizadas, la expresión di tibi dent quae uelis! (iii) presenta en las comedias plautinas un considerable nivel de variación léxica y morfosintáctica. Aparte de la variación en el orden de palabras (p. ej. tibi dent / dent tibi), se constatan fluctuaciones en la morfología verbal (dent / dabunt/ duint) $)^{22}$ o en la selección léxica (ueli[ti]s / optes/ exoptes; quae/ quaequomque). En consecuencia, es difícil determinar una versión canónica de la fórmula. Por añadidura, tampoco la función pragmática parece influir en la selección de la variante lingüística, puesto que las fórmulas de bendición propiamente dicha (i) o con fuerza ilocutiva secundaria gratulatoria (ii) presentan la misma variabilidad.

\section{i. bendición $(\mathrm{x} 2)$}

Tantum tibi boni di immortales duint quantum tu tibi optes (Pseud. 936) at di dabunt (Rud. 107)

${ }^{21}$ Las fórmulas de saludo en la comedia romana han sido objeto de estudio por parte de Forberg 1913, Echols 1950, Müller 1997, Poccetti 2010, Torrego 2013. El interesante trabajo de Letessier 2000 ofrece un buen ejemplo del significado interaccional (y dramatúrgico) que la construcción de la salutatio adquiere en los diálogos plautinos.

${ }^{22}$ La variación entre las formas de subjuntivo (dent) y de futuro (dabunt) existe en ambas fórmulas de saludo aquí analizadas (cf. di te ament/amabunt!). Por lo general, las gramáticas señalan que los dos paradigmas confluían en el latín arcaico, siendo intercambiables (véase Hofmann y Szantyr 1972, pp. 309-310), si bien las variantes de indicativo pueden indicar mayor grado de convicción (el llamado futuro aseverativo). 
ii. bendición/ acto gratulatorio (x6)

di tibi dent quaequomque optes (Asin. 46)

multa tibi di dent bona, quom ... (Poen. 208-209, *68723)

di deaeque uobis multa bona dent, quom ... (Poen. 667)

di dent tibi omnes quae uelis (Poen. 1055)

di dent quae uelis (Stich. 469)

iii. bendición/ saludo (x7)

di dent (tibi) quae uelis (Epid.6, Persa 483, Trin. 1152) ${ }^{24}$

dabunt di quae uelitis uobis (Asin. 623)

dabunt di quae exoptes (Persa 16)

di tibi dent quaequomque optes (Mil. 1038)

di duint tibi quaequomque optes (Trin. 436-437)

multa tibi di dent bona, quom ... (Poen. *687)

En el contenido proposicional de la fórmula, diferentes uerba optandi constituyen variantes expresivas: desde el neutro uelle hasta exoptare. Igual de significativo parece ser el uso del subjuntivo en la subordinada (quae[quomque] uelis / optes / exoptes), pese a que algunos comentaristas lo consideren un ejemplo de assimilatio modi ${ }^{25}$. En contra de esta interpretación, compárense los siguientes pares adyacentes:

(6a) SAG. Di deaeque me omnes perdant- /

PAE. Amicus sum, eueniant uolo tibi quae optas. (Persa 292-293)

23 La fórmula de Poen. 687 está recogida en dos lugares distintos dada su ambigüedad, señalada ya en el apartado anterior.

${ }^{24}$ La interpretación de Persa 483 es algo más problemática. El lenón Dórdalo no usa la fórmula di dent quae velis! en la réplica inmediata al saludo de su interlocutor, pese a que toda la secuencia funciona como una apertura dialógica. Por lo demás, el pasaje tiene un marcado carácter lúdico, si bien la interacción entre Dórdalo y Tóxilo no deja de ser algo enigmática (véase la explicación en el comentario de Woytek 1982).

25 Bennett 1910, pp. 305-314, trata de explicar - un tanto forzosamente-cada irregularidad del uso de subjuntivo en Plauto por una atracción «mecánica» de los modos en la oración principal. Por lo demás, Bertini 1986, ad loc., a la hora de comentar el pasaje de Asin. 45 (di tibi dent quaequomque optes), lo explica a través de la asimilación de modos, citando a Lindsay 1907, p. 66. El comentarista no matiza, sin embargo, que Lindsay 1907, p. 67, se decide por esta solución tras cierta vacilación. Más recientemente, De Melo 2007, p. 252, cuando se refiere a la fórmula de saludo di duint tibi, Philto, quaequomque optes (Trin. 436-7), concluye con seguridad que la forma de subjuntivo optes está influida por el arcaizante duint. 
(6b) Alc. Di me perdant-

MEL. Quodquomque optes, tibi uelim contingere. (Cist. 497)

En ambos casos el hablante expresa una gran desesperación a través de una automaldición (di me perdant!), que resulta cómicamente desautomatizada en boca de su interlocutor: en vez de una exclamación emotiva, la fórmula es entendida al pie de la letra como un acto optativo. Ahora bien, en (6a) el uso del indicativo (... quae optas) tiene carácter anafórico por referirse directamente al discurso precedente de su interlocutor. Melenis (6b), a su vez, resulta ser mucho más sarcástica y malévola, puesto que a través del uso del subjuntivo (quodquomque optes) quiere que se cumplan todos los deseos del joven Alcesimarco: tanto el que acaba de expresar, como los futuros.

Del mismo mecanismo parecen servirse las fórmulas de saludo que, sin excepción, se formulan en subjuntivo hipotético independiente. Para evidenciar el diferente tono de estas variantes, compárense los diálogos en (7) y (8), que que presentan intercambios entre un joven y un anciano. Las fórmulas iniciativas en ambos casos marcan un registro diafásico oficial con referencias en $3^{\mathrm{a}}$ persona, perteneciente al código escrito ${ }^{26}$. Sus respectivas respuestas, tal como podíamos esperar, también resultan formales, siendo por lo general el tono del par adyacente en (7) más elevado. El joven Lesbónico, dirigiéndose al anciano Filtón, escoge la forma arcaizante duint, lo cual podría interpretarse como su intento de adaptarse al registro del interlocutor ${ }^{27}$.

(7) PHIL. Erum atque servom plurumum Philto iubet / salvere, Lesbonicum et Stasimum.

LES. Di duint / tibi, Philto, quaequomque optes. quid agit filius? (Trin. 435-7)

(8) Lys. Charmidem socerum suom / Lysiteles salutat.

Char. Di dent tibi, Lysiteles, quae uelis. (Trin. 1051-2)

(9) $=(3)$ supra

26 Véanse las salutationes epistolares de Bacch. 734; Curc. 429-32; Persa 501-2.

27 De Melo 2007, pp. 247-8, apunta a esta posibilidad en la explicación de este uso concreto de un arcaísmo. Un ejemplo del fenómeno en cuestión, según el estudioso, se puede encontrar en el diálogo del joven Nicodemo con el anciano Dinia (Vid. 83-87). 
Aquí también habrá que mencionar la apertura dialógica paródica entre dos esclavos (9), comentada ya al principio de este artículo (véase también infra 18b). En suma, se puede constatar que en estas escenas de salutatio el bien recibido es devuelto: las expectativas mutuas de quien saluda y del saludado quedan satisfechas.

Otro caso es el de (10), donde el esclavo Epídico da la bienvenida a su colega Tesprión, cuando este acaba de regresar de una misión militar.

(10) Thes. Salue.

EPI. Di dent quae uelis. / uenire saluom gaudeo.

THEs. Quid ceterum?

EPI. Quod eo adsolet: / cena tibi dabitur. (Epid. 6-8)

El contexto de la interacción, por tanto, es muy específico: el equilibrio interpersonal deja terreno al complejo ritual de dar la bienvenida. El recién llegado ha de ser saludado con un ritual complejo: un saludo principal, la expresión de la alegría por la llegada (saluom [te] aduenire gaudeo), la pregunta por la salud y la invitación a comer, mientras que él mismo tiene pocas obligaciones para con su interlocutor. La fórmula de bendición usada por Epídico como respuesta a un simple salue!, por tanto, pone de manifiesto la falta de reciprocidad en la bienvenida, así como la falta de respuesta a la muestra de cortesía positiva formulada en términos de alegría por la llegada del interlocutor. Esto, a su vez, se comprueba en la otra parte de la apertura, cuando Tesprión tiene que recordar a su interlocutor cuál debería ser el subsiguiente paso conversacional (quid ceterum?).

Existen, por último, casos donde una interpretación literal del saludo (como un acto optativo) sirve como un recurso cómico. Así, tras romper lúdicamente las convenciones lingüísticas, se comprueba la existencia del mecanismo que fue base de su fijación. Este fenómeno humorístico (praeter exspectationem) viene recogido en los pasajes de (11) y (12):

(11) MIL. Pulcher, salve. /

PYR. Meum cognomentum commemoravit. Di tibi dent quaequomque optes. /

MitPh. Tecum aetatem exigere ut liceat-

PYr. Nimium optas.

MiLPH. Non me dico, / sed eram meam, quae te demoritur.

(Mil. 1037-1040) 
(12) LIB. Philaenium, salue.

PHIL. Dabunt di quae uelitis uobis. /

LIB. Noctem tuam et uini cadum uelim, si optata fiant. /

ARG. Verbum caue faxis, uerbero.

LiB. Tibi equidem, non mihi opto. (Asin. 623-635)

Algún matiz gratulatorio de la fórmula se podría detectar en (11), dado que el soldado fanfarrón está reaccionando a las blanditiae de Milfidipa, quien lisonjeramente le llama «guapo» en la identificación. En la boca de Filenia (12), a su vez, la bendición no parece desempeñar otra función más allá de un contrasaludo ${ }^{28}$.

Como conclusión, cabe señalar que los saludos di tibi dent quae uelis! y sus variantes aparecen en conversaciones de cierta formalidad, sobre todo, entre dos ciudadanos cultos (7), (8). Siempre que en la interacción aparece un personaje de clase baja (esclavo, sirvienta o lenón), es evidente el empleo paródico o irónico de la fórmula (9)-(12), que a veces consigue marcar distancias con el interlocutor. Así habría que explicar el único uso de este tipo de saludo en el lenguaje femenino en (12). Otro contexto recurrente es el ritual de la bienvenida después de un largo y peligroso viaje (9), (10): aquí también nos encontramos con rasgos de un contacto más bien formal y ceremonioso. Conviene subrayar, por último, que ninguno de estos casos excluye definitivamente la interpretación como acto gratulatorio de la bendición, apreciación que resulta reforzada por la posición reactiva de la fórmula di tibi dent quae uelis! (y sus variantes) en todas los testimonios recogidos. Ello, añadido a la escasa fijación formal, hace que su funcionamiento como respuesta a un saludo no difiera sustancialmente desde un punto de vista nocional de las fórmulas análogas para expresar agradecimiento. Ahora bien, solamente en (8), (9) y (11) el contexto inmediato de la apertura dialógica admite una doble interpretación de su función como fórmula de contrasaludo y expresión gratulatoria.

${ }^{28}$ Si bien dabunt di quae uelitis uobis! usado por Filenia es un ejemplo de fórmula de contrasaludo elaborada, la intención de la joven cortesana es realmente marcar distancia con los dos interlocutores de baja condición social, que resultan molestos. Véase Forberg 1913, p. 31: «Verba puellae dolentis 'dabunt di quae uelitis uobis' hoc fere significant: 'Quid uestra salutatio iuuat nos, qui periimus? Nihil nobis uobiscum est. Dabunt di quae uelitis uobis.' Itaque haec formula, etsi non incommode dicta est, tamen uim repellendi in se habet.» 
2. Di te ament!

La fórmula conversacional di te ament! se encuentra únicamente en las comedias de Plauto, donde asume las siguientes variantes:

i. di te ament (Aul. 183, Bacch. 457, Curc. 455, Most. 341, 806, 1130, Poen. 751, Pseud. 1294, Rud. 1303)

ii. $\quad$ di ament te (Persa 16)

iii. di te / me amabunt (Men. 278, Persa 205)

iv. di te bene ament (Capt. 138), di te ament plurumum (Most. 717)

La versión canónica (i) di te ament (x9) presenta variantes morfológicas con formas de futuro aseverativo ${ }^{29}$ o modificada con adverbios intensificadores (iv) bene o plurumum ${ }^{30}$. En cuanto al orden de constituyentes no marcado $(\mathrm{S}=d i ; \mathrm{O}=t e ; \mathrm{V}=$ ament), tan solo en una variante (ii) se encuentra un esquema SVO, aparentemente sin cambios para su significado interaccional. La mayor extensión léxica (v) acompaña siempre a un empleo cómico. Por lo demás, Lindsay 1961, p. 156, apunta que, en el habla cotidiana, el pronombre personal te debía de ser proclítico: de ahí su frecuente localización al principio del verso ${ }^{31}$, donde experimenta un ictus natural (dit'ament).

Asimismo, cabe presentar la distribución de la fórmula dentro del par adyacente. En la mayor parte de los casos aparece en posición reactiva (13)-(16), regularmente con la identificación del destinatario (p. ej. di te ament, Megadore). Entre los usos más regulares se puede enumerar los siguientes pasajes:

(13) Meg. Saluos atque fortunatus, Euclio, semper sies. /

Euc. Di te ament, Megadore. (Aul. 182-183)

(14) CAL. Eu, Philolaches, / salue, amicissime mi omnium hominum. / PHILo. Di te ament. accuba, Callidamates. (Most. 339-341)

(15a) Phil. Saluos sis, Mnesiloche, saluom te aduenire gaudeo. / MNE. Di te ament, Philoxene. (Bacch. 456-457)

${ }^{29}$ Véase supra n. 22.

${ }^{30}$ Los adverbios intensificadores modifican también otras fórmulas de saludo o despedida: véanse salve multum! (p. ej. Men. 775, Persa 739, Poen. 1050) o bene uale! (p. ej. Amph. 499, Asin. 606, Capt. 452, Cist. 113).

${ }^{31}$ Aul. 182-183, Bacch. 456-457, Most. 339-341, 805-806. 
(15b) Simo. Saluom te aduenisse peregre gaudeo, Theopropides. /

THeo. Di te ament. (Most. 805-806)

(15c) CAL. ... iubeo te saluere et saluos cum aduenis, Theopropides, / peregre, gaudeo. hic apud nos hodie cenes, sic face. / Theo. Callidamates, di te ament. de cena facio gratiam. (Most. 1128-1130)

De acuerdo con lo que sugerían las palabras de Agorastocles, recogidas en (2), la fórmula di te ament!, igual que su equivalente di tibi dent quae uelis!, habría de ser considerada un mecanismo potencial de cortesía positiva. Efectivamente, en los pasajes localizados predominan los contextos marcadamente apreciativos: conversaciones amigables entre vecinos $(13)^{32}$, entre jóvenes durante un banquete (14) y en forma de una ceremoniosa bienvenida, entre dos miembros de la élite, después de un largo viaje a casa (15a-c).

En boca de los personajes de extracción baja (esclavo, parásito, lenón), la fórmula implica un efecto paródico o constituye un uso estratégico de una salutatio de tono elevado.

(16a) Heg. Quis hic loquitur?

ERG. Ego, qui tuo maerore maceror, / macesco, consenesco et tabesco miser; / ossa atque pellis sum misera ... macritudine; / neque umquam quicquam me iuuat quod edo domi: / foris aliquantillum etiam quod gusto id beat. /

Heg. Ergasile, salue.

ERG. Di te bene ament, Hegio. (Capt. 133-138)

(16b) LAB. Adulescens, salve.

GRI. Di te ament cum irraso capite.

LAB. Quid fit? /

GRI. Verum extergetur.

LAB. Vt uales?

GRI. Quid tu? Num medicus, quaeso, es? (Rud. 1303-1304)

32 En el caso de la salutatio en (13) disponemos del testimonio de Euclión de que los turnos anteriores de su interlocutor incluyen marcas de la cortesía positiva (Aul. 185: Euc. ... iam illic homo aurum scit me habere, eo me salutat blandius). Podemos, por tanto, dar por supuesto que el contrasaludo del mismo anciano se ha adecuado a este tono: de ahí la elección de la fórmula di te ament! 
Un ejemplo de lo último encontramos en (16a), donde el parásito Ergásilo se autoidentifica como un sirviente empático (ego, qui tuo maerore maceror) y, a la vez, un compañero hambriento (ossa atque pellis sum misera) del hijo de su interlocutor. El rebuscado saludo di te bene ament! formaría parte de la estrategia usada por el esclavo para sonsacarle al anciano Hegión una invitación a cenar a cambio de consuelo y compasión (cf. Capt. 172-173: ERG. ... ita di deaque faxint. sed num quo foras / uocatus 〈es〉 ad cenam?). E1 uso paródico se hace visible en el turno de Gripo (16b), quien a la fórmula artificiosa añade una identificación sarcástica y una postura conversacional agresiva en el resto del diálogo ${ }^{33}$.

El siguiente pasaje (17a) es un ejemplo de una interacción formal (en un contexto financiero) entre dos personajes de extracción baja. En el ámbito profesional (entre un leno ${ }^{34}$ Capadocio y un tarpezita Licón) los interlocutores se tratan como iguales según su propia percepción, sin recurrir al sarcasmo ni a la agresión verbal, si bien su salutatio no contiene otras marcas de cortesía positiva como en (13)-(15). Por lo tanto, di te ament! funciona aquí como un mecanismo de comportamiento político en contextos formales.

(17a) LyCO ... leno, salue.

CAP. Di te ament. /

LYCO. Quid hoc quod ad te uenio?

CAP. Dicas quid uelis (Curc. 455-456)

(17b) CyL. ... Menaechme, salue.

Sos. Di te amabunt quisquis 〈es〉. /

CyL. Quisquis d-*** 〈quis〉 ego sim?

Sos. Non hercle vero. (Men. 278-280)

Este uso formal pero no propiamente cortés reaparece en (17b), donde el cocinero Cilindro intenta entablar conversación con Sósicles (Menecmo II), a quien ha confundido con Menecmo I, el amante de su ama. De ahí que use el saludo más corriente e informal (salue!) acompañado de una identifica-

${ }^{33}$ Véase el comentario de Fay 1993, ad loc.: «Whatever the point of the insult (sc. cum irraso capite) was, at quid fit? Labrax swallows it, and tries again to enter into amicable conversation».

${ }^{34}$ Dickey 2002, p. 237, apunta que el vocativo leno! en la comedia casi siempre conlleva un matiz peyorativo (p. ej. Curc. 525, 715), si bien este pasaje de Curc. 455, según reconoce la autora, no presenta evidentes marcas de descortesía. 
ción nominal (Menaechme). Su interlocutor, llamado por un nombre que no es el suyo, cumple igualmente con el ritual de la apertura dialógica, pero su elección de la fórmula de bendición, posiblemente, ha de ser interpretada por parte del parásito como un gesto de distanciamiento ${ }^{35}$. A saber, un saludo más elaborado $\mathrm{y}$, potencialmente, más cortés funciona aquí como una indicación del rechazo del contacto que se pretende cercano, según trasluce salue!

Si tomamos en cuenta que, en todos estos pares adyacentes de la salutatio, la fórmula di te ament! ocupa la posición reactiva, podría reconocerse en su uso cierto contenido residual de agradecimiento. Ahora bien, recordemos que en la comedia romana di te ament!, a diferencia del otro saludo que incluye a los dioses di tibi dent quae uelis!, no se atestigua con más funciones discursivas que la de saludo. Las bendiciones con más semejanzas léxicas y semánticas (Pseud. 934: Iuppiter te mihi seruet; Mil. 231-32: te Iuppiter / bene amet), no obstante, pueden ser empleadas con sentido gratulatorio. Este significado adicional de la salutatio en cuestión sería especialmente reconocible en las réplicas a las expresiones de alegría del tipo saluom te (ad)uenire gaudeo! (15) y en los saludos e identificaciones emotivos y menos convencionales (13), (14). Por lo demás, el matiz de agradecimiento seguramente no sería tan perceptible en di te ament! como en di tibi dent quae uelis!, tal y como se ha defendido en el apartado anterior.

Otra particularidad de esta fórmula consiste en su capacidad de ocupar aunque solamente en tres variantes- una posición iniciativa. Esta aparente irregularidad fue señalada también por Forberg 1913, pp. 27-28, quien, sin embargo, no le da ninguna explicación, atribuyéndola a la libertad del estilo plautino ${ }^{36}$. Por lo demás, este autor insiste en tratar tanto di te ament! como di tibi dent quae uelis! como fórmulas reactivas (formulae resalutandi), señalando su origen en las expresiones de agradecimiento. A nuestro modo de ver, no obstante, esta variación va más allá de una irregularidad estilística, lo que demuestra claramente un paso ulterior en el proceso referido aquí como repragmaticalización.

Veamos estos ejemplos. Con un di te ament! iniciativo Tranión se dirige al viejo Simón (18a), mientras que el esclavo Tóxilo inicia una conversación

35 Véase supra n. 28.

36 Forberg 1913, pp. 27-28: «Negari nequit his locis formula nostra salutari, non resalutari. Quod maxime mirum est. Sed videmus usum Plautinum hic etiam liberum esse.» 
amistosa con su colega Sagaritsión (véase ejemplo (3) reproducido aquí como (18b)):

(18a) TRA. ... di te ament plurumum, Simo. /

Simo. Saluos sis, Tranio.

TRA. Vt uales?

Simo. Non male. / quid agis?

TRA. Hominem optumum teneo. (Most. 717-719)

(18b) Tox. O Sagaristio, di ament te.

SAG. O Toxile, dabunt di quae exoptes. / ut uales?

Tox. Vt queo.

SAG. Quid agitur?

Tox. Viuitur. (Persa 16-17)

Es significativo notar que estos casos no difieren en nada de los que acabamos de analizar (con la fórmula de bendición en la réplica: (13)-(17)). Ambas aperturas (18a-b) transcurren de una manera incluso más armónica, tanto en lo que atañe a la simetría de los actos (saludo + identificación nominal), como en la introducción de small talk preliminar (ut uales?, quid agis / agitur?) ${ }^{37}$.

(18c) PSEU. Vir malus uiro / optumo obuiam it. /

Simo. Di te ament, Pseudole.

Pseu. Hae!

Simo. I in malam crucem. /

PsEu. Cur ego afflictor?

Simo. Quid tu, malum, in os igitur mi ebrius irructas?

(Pseud. 1292-1295)

Algo totalmente opuesto ocurre en un último ejemplo de di te ament! iniciativo (18c), donde un Pséudolo borracho y triunfante, hacia el final de la obra, es saludado por su amo Simón. De hecho, podemos acceder directamente a las intenciones del anciano Simón que subyacían en su manera de empezar el diálogo:

${ }^{37}$ La secuencia de small talk es lo que el antropólogo Malinowski 1923, pp. 314-315, identificaba al principio del diálogo como la comunión fáctica: «a type of speech in which ties of union are created by a mere exchange of words». Aquí se entiende este fenómeno como un tema preliminar convencional (p.ej. la salud del interlocutor), que sigue el intercambio de saludos y precede la introducción del primer tema de relevancia (cf. n. 10). 
(18d) Simo. ... cogito, saeuiter blanditerne adloquar. / sed me hoc uotat uim facere nunc / quod fero, si qua in hoc spes sita est mihi. (Pseud. 1290-1291a)

El senex duda de qué tipo de actitud le conviene más en la interacción con su esclavo, para no tener que pagarle el merecido premio. Finalmente, decide tratar con su interlocutor amablemente (blanditer adloquar), llevado por la esperanza de que, así, conseguirá ablandar al arrogante Pséudolo. La única respuesta del esclavo es un eructo directo en la cara. El tono elevado del saludo, por tanto, es frustrado por su reacción grosera y puramente fisiológica.

Para terminar, hemos reservado algunos casos de desautomatización de la fórmula, que es un fenómeno típicamente plautino e, indirectamente, da fe de su estatus de cliché lingüístico con alto grado de pragmaticalización, si bien su contenido proposicional sigue siendo transparente. Son las siguientes aperturas de carácter lúdico:

(19a) Soph. Paegnium, deliciae pueri, salve. quid agis? ut uales? /

PAE. Sophoclidisca, di ... me amabunt.

Soph. Quid me?

PAE. Vtrum hercle <illis lubet〉;/ sed si ut digna es faciant, odio hercle habeant et faciant male./

Soph. Mitte male loqui. (Persa 204-207)

(19b) MiL. Saluos sis, Synceraste.

Syn. O Milphio, / di omnes deaeque ament.

MiL. Quemnam hominem?

Syn. Nec te nec me, Milphio: / neque erum meum adeo.

MıL. Quem ament igitur?

Syn. Aliquem, dignus qui siet. / nam nostrorum nemo dignust. (Poen. 858-861)

En (19a) Pegnio consigue el efecto cómico cuando, al saludar a Sofoclidisca, convierte la expresión de respuesta al saludo de su interlocutora en una autobendición ${ }^{38}$. Sincerasto, a su vez, recurre a una fórmula incompleta (19b), dejando en suspenso todo el acto del saludo para constatar, al final, que ninguno de los interlocutores se merece la bendición divina.

${ }^{38}$ Algo parecido ocurre en Poen. 869-870, Rud. 105-109. 


\section{Conclusiones}

En el presente artículo nos hemos ocupado de dos fórmulas conversacionales, rutinizadas y especializadas funcionalmente, que, a resultas de un proceso de pragmaticalización, han dejado de lado parte de su contenido proposicional para desempeñar funciones de tipo pragmático (y discursivo). En particular, nuestra atención se ha centrado en un paso más avanzado de este proceso, a saber: los casos en los que se desarrollan empleos derivados de las fórmulas preexistentes, que implican una posible redistribución (adicional o exclusiva) de las funciones pragmáticas básicas. Siguiendo a Claridge y Arnovick 2010, p. 183, creemos razonable reconocer como diferente al de pragmaticalización un proceso que afecta a elementos que tienen función pragmática previa, que denominamos repragmaticalización (pragmaticalización secundaria) o, más exactamente, redistribución de la función pragmática ${ }^{39}$. Este planteamiento se basa en la presuposición de que algunos actos comunicativos vinculados con ciertas formas lingüísticas rutinizadas constituyen la base de otras funciones pragmáticas de alguna manera dependientes de su fuerza ilocutiva original. Así, hemos visto que los actos de bendición en el lenguaje de la comedia romana llegaron a funcionar -entre otras posibilidades- como una expresión de gratitud. Existen fuertes indicios de que, partiendo de este significado interaccional, las fórmulas di te ament! y di tibi dent quae uelis!, presentes únicamente en Plauto, se especializaron en la función de respuesta a un saludo.

Por otro lado, tras analizar todo el material recogido podemos presentar algunas conclusiones de índole teórica acerca del proceso de repragmaticalización. Si recurrimos a los casos de las expresiones rutinizadas, aquí comentadas, como rasgos que caracterizan un proceso de redistribución de la función pragmática podemos reconocer un mayor grado de:

I. desemantización

II. desinstitucionalización

III. fijación léxica (paradigmática)

IV. fijación sintagmática (a nivel global)

V. libertad posicional (a nivel local)

${ }^{39}$ Claridge y Arnovick 2010, p. 183, prefieren el término «discursivización» (ing. discursisation), que nos parece poco transparente y demasiado cercano al término «discursificación» empleado por Foucault. 
Las fórmulas de bendición, especializadas primero en la fuerza ilocutiva gratulatoria, en su nueva función como saludo sufren una desemantización ulterior (I): la total desvinculación del contexto religioso. Si bien el origen de las fórmulas y su significado proposicional, incluso sincrónicamente, no dejan de ser transparentes, parece razonable pensar que los saludos del tipo $d i$ te ament! perdieron el carácter performativo de una bendición propiamente dicha, convirtiéndose en una convención de cortesía. Cabe matizar, sin embargo, que el contenido religioso, por decirlo de alguna manera, mantiene una presencia latente, al alcance de los usuarios, lo cual queda demostrado por los casos de desautomatizaciones lúdicas de las fórmulas, p. ej. (11), (12), (19a-b). Dada su transparencia léxica, cada saludo expresado mediante una bendición puede fácilmente recuperar su sentido proposicional.

Con la desemantización se relaciona, asimismo, el diferente grado de desinstitucionalización (II). El contexto gratulatorio se ha debilitado, dejando de ser palpables los conceptos de favor y deuda contraída: dentro del par adyacente de la salutatio, las prestaciones recíprocas consisten en declaraciones de beneuolentia simbólicas y rutinarias. Ahora bien, tal como hemos señalado, la fórmula di tibi dent quae uelis! sigue siendo ambigua, debido tanto a su fijación en el turno reactivo, como al hecho de que uno de sus testimonios (5) puede considerarse un contexto puente entre el acto gratulatorio y el saludo.

Tampoco el parámetro de la fijación paradigmática (III) demuestra que la mencionada expresión haya sufrido una fuerte repragmaticalización. Con todo, se ha de notar cierta tendencia hacia la fijación léxica en la función de saludo: la variante di tibi dent quae uelis! parece algo más frecuente, mientras que, en la nueva función, la fórmula prescinde de la frase subordinada causal (quom...), en la que se suele explicitar el objeto de gratitud en los empleos puramente gratulatorios. La fijación léxica de di te ament!, por otro lado, parece casi absoluta, alejándose significativamente de otras variantes de las expresiones de bendición. Hay que señalar, no obstante, que el material a nuestra disposición es escasísimo y, desafortunadamente, no podemos recurrir a ningún contexto de transición. Lo que nos ofrece el lenguaje plautino es una fórmula de saludo totalmente especializada: di te ament! A pesar de la sistematicidad con la que aparece, su función pragmática debió de sufrir una redistribución paradigmática hasta cierto punto similar a la de otras rutinas lingüísticas de bendición (como di tibi dent quae uelis!).

Ahora bien, en el caso de di te ament! ha operado el último parámetro: la mayor libertad sintagmática dentro del par adyacente (IV). Si bien ambas fór- 
mulas, como nuevos mecanismos de saludo, sufren una redistribución posicional a nivel global (en la apertura dialógica), únicamente di te ament! puede aparecer tanto en la réplica como en la iniciación del par adyacente de la salutatio. Así, en la evolución de esta expresión rutinaria, visiblemente más especializada en la nueva función discursiva, se deja notar una tendencia a extender su uso como saludo universal (en cuanto a la posición en el par adyacente) a costa de ciertos matices adicionales (p. ej. gratulatorios), tal y como se representa en la Tabla 2. Claro está, este último parámetro no es en absoluto obligatorio y únicamente concierne a unidades pragmáticas que, aparte de la redistribución de su función interpersonal, hayan sufrido un cambio de categoría.

Tabla 2. Fórmulas de saludo más frecuentes en Plauto según su posición dentro del par adyacente.

\begin{tabular}{|c|c|c|c|}
\hline \multirow{2}{*}{ Fórmula } & \multicolumn{2}{|c|}{ posición en el par adyacente } & \multirow{2}{*}{ Total } \\
\hline & iniciación & réplica & \\
\hline salue! & 59 & 27 & 86 \\
\hline di te ament! & 3 & 13 & 16 \\
\hline di tibi dent quae uelis! & - & 7 & 7 \\
\hline iubeo te saluere! & 10 & - & 10 \\
\hline
\end{tabular}

Los dos procesos de pragmaticalización (primaria) y repragmaticalización se pueden considerar como un continuum de desemantización, desinstitucionalización y desarrollo de contenidos pragmáticos.

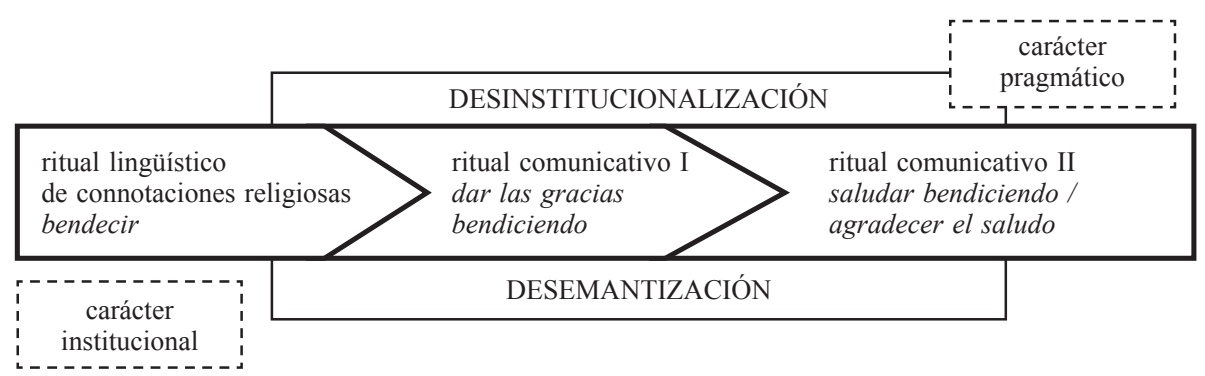

Para terminar, conviene matizar que este mecanismo de redistribución de la función pragmática concierne principalmente a las fórmulas de bendición reanalizadas como saludos. Incluso las mismas expresiones aquí tratadas, pese a tener un origen y un empleo discursivo similares, difieren bastante en cuanto a los principales parámetros apuntados (I-V). Aun así, entre las con- 
clusiones se puede encontrar muchos puntos en común con las generalizaciones de Claridge y Arnovick (2010). A nuestro modo de ver, reconocer un caso de posible repragmaticalización e intentar explicar su motivación, desde el punto de vista institucional (socio-cultural) y situacional (pragmático), entraña un mayor rigor científico y, a la par, facilita una información más completa acerca de las rutinas comunicativas reflejadas en las comedias de Plauto.

\section{BiBLIOGRAFÍA}

Bennett, Ch. E. 1910: Syntax of early Latin. Vol. 1. The verb, Boston.

Benveniste, É. 1966: «Don et échange dans le vocabulaire indo-européen», en íd., Problèmes de linguistique générale I, París, pp. 315-326.

Berger, Ł. 2016: Otwarcie dialogowe w komediach Plauta, Poznań (tesis doctoral). Bertini, F. 1968: Plauti Asinaria cum commentario exegetico. Pars prior: Prolegomena et Textus; pars altera: Commentarium et Indices, Génova.

Brown, P. y Levinson, S. C. 1987: Politeness: Some universals in language usage, Cambridge.

Burton, P. J. 2004: «Amicitia in Plautus: A study of Roman friendship processes», AJPh 125, pp. 209-243.

Claridge, C. y Arnovick, L. 2010: «Pragmaticalisation and Discursisation», en Jucker, A. H. y Taavitsainen, I. (eds.), Historical pragmatics, Berlín - Nueva York, pp. 165-192.

Coulmas, F. 1981a: «Introduction: Conversational Routine», en íd. (ed.), Conversational Routine. Explorations in Standarized Communication Situations and Prepatterned Speech, La Haya, pp. 1-17.

Coulmas, F. 1981b: «Poison to Your Soul. Thanks and Apologies Contrastively Viewed», en íd. (ed.), Conversational Routine. Explorations in Standarized Communication Situations and Prepatterned Speech, La Haya, pp. 69-91.

De Melo, W. 2007: The Early Latin Verb System: Archaic Forms in Plautus, Terence, and Beyond, Oxford.

De Melo, W. (ed.) 2011-2013: Plautus. Comedies. 5 vols, Cambridge, MA.

Degand, L., y Evers-Vermeul, J. 2015: «Grammaticalization or pragmaticalization of discourse markers? More than a terminological issue», Journal of Historical Pragmatics 16 (1), pp. 59-85.

Dickey, E. 2002: Latin Forms of Address, Oxford.

Duranti, A. 1997: «Universal and Culture-Specific Properties of Greetings», Journal of Linguistic Anthropology 7, pp. 63-97.

Echols, E. C. 1950: «The Quid-Greeting in Plautus and Terence», CJ 45 (4), pp. 188-190. 
Fay, H. C. (ed.) 1993: Plautus. Rudens, Londres.

Ferguson, Ch. A. 1981: «The Structure and Use of Politeness Formula», en Coulmas, F. (ed.), Conversational Routine. Explorations in Standarized Communication Situations and Prepatterned Speech, La Haya, pp. 21-35.

Forberg, M. 1913: De salutandi formulis Plautinis et Terentianis, Weidae Thuringorum.

Goffman, E. 1955: «On Face-Work», Psychiatry 18 (3), pp. 213-231.

Goffman, E. 1971: Relations in Public: Microstudies of the Social Order, Londres.

Goody, E. 1972: “'Greeting', 'begging' and the presentation of respect», en La Fontaine, J. S. (ed.), The Interpretation of Ritual: Essays in Honour of A.I. Richards, Londres, pp. 39-72.

Griffe, M. 1985: «Ita me di ament (ut...). Une formule de serment en latin ancien», Lalies 7, pp. 289-298.

Hanson, J. A. 1959: «Plautus as a Source Book for Roman Religion», TAPhA 90, pp. 48-101.

Hoffmann, M. 1983: «Conversational Openings in the Comedies of Plautus», en Pinkster, H. (ed.). Latin Linguistics and Linguistics Theory, Amsterdam - Filadelfia, pp. 217-226.

Hofmann, J. B., y Szantyr, A. 1972: Lateinische syntax und stilistik, vol. 2, Múnich.

Laver, J. 1975: «Communicative function of phatic communion», en Kendon A., Harris, R. S. y Key, M. R. (eds.), Organization of Behavior in Face-to-Face Interaction, La Haya, pp. 215-238.

Letessier, P. 2000 : «La salutatio chez Plaute. Adaptation ludique d'un rituel social», Lalies 20, pp. 151-163.

Levinson, S. C. 1983: Pragmatics, Cambridge.

Lindsay, W. M. 1961 (ed.): The Captivi of Plautus, Cambridge.

Lindsay, W. M. 1907: Syntax of Plautus, Oxford.

Malinowski, B. 1923: «The Problem of Meaning in Primitive Languages», en Ogden, C. K. y Richards, I. A. (eds.), The Meaning of Meaning, pp. 296-336.

Monserrat Roig, C. 2015: «Otras perspectivas para el análisis lingüístico de Plauto: los vocativos insultantes en la reacción conversacional», Minerva 28, pp. 133-161.

Müller, R. 1997: Sprechen und Sprache. Dialoglinguistische Studien zu Terenz. Heidelberg.

Pavlidou, T-S. 2014: «Phases in discourse», en Schneider, K. P. y Barron, A. (ed), Pragmatics of discourse, vol. 3, Berlín - Boston, pp. 353-383.

Poccetti, P. 2010: "Greeting and farewell expressions as evidence for colloquial language: between literary and epigraphical texts», en Dickey, E. y Chahoud, A. (eds.), Colloquial and Literary Latin, Cambridge, pp. 100-126.

Ronan, P. 2015: «Categorizing expressive speech acts in the pragmatically annotated SPICE Ireland corpus», ICAME Journal 39 (1), pp. 25-45. 
Torrego Salcedo, M. E. 2013: «Iubeo saluere: una forma de saludo con directivo léxico», en Beltrán, J. A. et al. (eds.), Otium cum dignitate, Zaragoza, pp. 173-184.

Unceta Gómez, L. 2010: «La expresión del agradecimiento en la comedia latina», en Anreiter, P. y Kienpointner, M. (eds.), Latin Linguistics today. Proceedings of the XVth International Colloquium on Latin Linguistics, Innsbruck, pp. 625-637.

Verboven, K. 2011: «Friendship among the Romans», en Peachin, M. (ed.), The Oxford Handbook of Social Relations in the Roman World, Oxford, pp. 404-421. Watts, R. J. 2003: Politeness, Cambridge.

Woytek, E. (ed.) 1982: T. Maccius Plautus: Persa. Einleitung, Text und Kommentar, Viena.

Fecha de recepción de la primera version del artículo: 16/09/2016

Fecha de aceptación: 14/12/2016

Fecha de recepción de la version definitiva: 11/02/2017 